\title{
Complex Genetic System Involved in Fusarium Ear Rot Resistance in Maize as Revealed by GWAS, Bulked Sample Analysis, and Genomic Prediction
}

\author{
Zifeng Guo, ${ }^{1,2}$ Cheng Zou, ${ }^{1}$ Xiaogang Liu, ${ }^{1}$ Shanhong Wang, ${ }^{1}$ Wen-Xue Li, ${ }^{1}$ Dan Jeffers,,${ }^{3,4}$ Xingming Fan, ${ }^{4}$ Mingliang Xu, ${ }^{2}$ \\ and Yunbi $\mathrm{Xu}^{1,3,5,6, \dagger}$ \\ ${ }^{1}$ Institute of Crop Science/International Maize and Wheat Improvement Center China, Chinese Academy of Agricultural Sci- \\ ences, Beijing 100081, China \\ ${ }^{2}$ National Maize Improvement Center of China, China Agricultural University, Beijing 100193, China \\ ${ }^{3}$ International Maize and Wheat Improvement Center, El Batan, Texcoco, CP 56130, México \\ ${ }^{4}$ Institute of Food Crops, Yunnan Academy of Agricultural Sciences, Kunming 650200, China \\ ${ }^{5}$ International Maize and Wheat Improvement Center China Specialty Maize Research Center, Shanghai Academy of Agricul- \\ tural Sciences, Shanghai 201400, China \\ ${ }^{6}$ International Maize and Wheat Improvement Center China Tropical Maize Research Center, Foshan University, Foshan \\ 528231, China
}

\begin{abstract}
Fusarium ear rot (FER) caused by Fusarium verticillioides is one of the most prevalent maize diseases in China and worldwide. Resistance to FER is a complex trait controlled by multiple genes highly affected by environment. In this paper, genome-wide association study (GWAS), bulked sample analysis (BSA), and genomic prediction were performed for understanding FER resistance using 509 diverse inbred lines, which were genotyped by 37,801 high-quality single-nucleotide polymorphisms (SNPs). Ear rot evaluation was performed using artificial inoculation in four environments in China: Xinxiang, Henan, and Shunyi, Beijing, during 2017 and 2018. Significant phenotypic and genetic variation for FER severity

(1_226233417 on chromosome 1 and 10_14501044 on chromosome 10) were associated at threshold of $2.65 \times 10^{-7}[-\log (0.01 / 37,801)]$. Using BSA, resistance quantitative trait loci were identified on chromosomes 3 , $4,7,9$, and 10 at the $90 \%$ confidence level and on chromosomes 3 and 10 at the $95 \%$ confidence level. A key region, bin 10.03, was detected by both GWAS and BSA. Genomic prediction for FER resistance showed that the prediction accuracy by trait-related markers was higher than that by randomly selected markers under different levels of marker density. Marker-assisted selection using genomic prediction could be an efficient strategy for genetic improvement for complex traits like FER resistance.
\end{abstract} was observed, and FER resistance was significantly correlated among the four environments with a generalized heritability of 0.78 . GWAS identified 23 SNPs that were associated with FER resistance, 2 of which
Keywords: bulked sample analysis, Fusarium verticillioides, genomewide association study, genomic selection, maize
Maize ear rots occur in all maize-producing areas worldwide and can be caused by many pathogens, including $>20$ fungi, such as Fusarium graminearum, Fusarium verticillioides, Aspergillus flavus, Penicillium spp., Cladosporium spp., and Trichothecium roseum (Görtz et al. 2008; Zummo and Scott 1990). The two most important fungi worldwide are $F$. verticillioides (synonym Fusarium moniliforme Sheldon) that causes Fusarium ear $\operatorname{rot}(\mathrm{FER})$ and $F$. graminearum that causes Gibberella ear rot (Mesterházy et al. 2012; Munkvold 2003). In China, FER is widely distributed in major maize-producing areas, causing serious harm to maize production (Duan et al. 2015). FER not only causes direct yield loss but also, produces fumonisins, a group of mycotoxins that affect grain quality. Fumonisins cause

\section{${ }^{\dagger}$ Corresponding author: Y. Xu; y.xu@cgiar.org}

Funding: This research is supported by National Key Research and Development Program of China grants 2017YFD0101201 and 2016YFD0101201 and Central Public-interest Scientific Institution Basal Research Fund (Y2020PT20) and Agricultural Science and Technology Innovation Program (ASTIP) of Chinese Academy of Agricultural Sciences (CAAS) grant CAAS-XTCX2016009. Research activities of the International Maize and Wheat Improvement Center staff were supported by the Bill and Melinda Gates Foundation and the CGIAR Research Program MAIZE.

*The $\boldsymbol{e}$-Xtra logo stands for "electronic extra" and indicates that eight supplementary figures and five supplementary tables are published online.

The author(s) declare no conflict of interest.

Accepted for publication 17 January 2020.

(C) 2020 The American Phytopathological Society serious health problems in animals that consume contaminated grain, and they are indirectly linked with adverse health effects in humans. High rates of esophageal cancer occur in the regions with high levels of fumonisins in corn (Munkvold and Desjardins 1997), and the highest fumonisin $B_{1}$ was observed in China (Placinta et al. 1999). Resistance to FER in maize is a complex trait that is controlled by many genes, each with minor effects, and it is strongly influenced by environmental conditions (Jeffers 2003; Rose et al. 2016). Host resistance is the most effective method to control FER, and many commercial maize hybrids lack adequate resistance to FER (Balconi et al. 2014; Bush et al. 2004; Mesterházy et al. 2012). The localizations of the main quantitative trait loci (QTL) and single-nucleotide polymorphism (SNP) markers for FER resistance are inconsistent or even contradictory in different studies (Lanubile et al. 2017). Many sources of resistance to FER have been identified in maize, but it is difficult to transfer resistance alleles from the donor lines to commercial breeding lines owing to many minor effect QTL for resistance. The heritability of individual plants against FER is relatively low, but the average resistance from repeated parental and diversity group studies was moderately or highly heritable (Robertson-Hoyt et al. 2006). Empirical studies have shown that phenotypic selection and marker-assisted selection (MAS) for FER resistance are effective (Eller et al. 2010).

Linkage mapping has been used to locate genomic regions related to maize FER resistance. However, it depends on biparents with large differences for FER resistance to develop segregating populations. Because segregating individuals are genotyped with only a limited number of random markers, the probability is low that these markers will include or be close to the functional genes (Yan and Warburton 2011). Several QTL for FER resistance have been identified using different maize biparental populations (Chen et al. 2012; Ding et al. 2008; Maschietto et al. 2017; Pérez Brito et al. 2001; Robertson-Hoyt et al. 2006), but they only can explain a small 
fraction of the phenotypic variation, with inconsistent results across populations and environments. Because linkage mapping is not only tedious but also, time consuming, genome-wide association study (GWAS) has been used as an alternative strategy in genetic mapping of complex traits in plants. In addition, GWAS can use germplasm accessions and their historical phenotypic data to explore all recombination events and mutations with high resolution ( $\mathrm{Yu}$ and Buckler 2006). Maize has abundant genetic and phenotypic diversity (Gore et al. 2009). Compared with other species, it has faster linkage disequilibrium (LD) decay and is very suitable for GWAS (Yan and Warburton 2011). GWAS has been widely used for genetic mapping of complex traits, including FER resistance (Chen et al. 2016; Coan et al. 2018; Zila et al. 2013, 2014).

However, GWAS analyzes all individuals collected from sample populations, and thus, it needs more research funds. Compared with GWAS, bulked sample analysis (BSA) only chooses the individuals with extreme phenotypes to form pooled samples for genotyping, thus reducing the cost and scale of the analysis (Zou et al. 2016). Traditional bulked segregating analysis uses only biparental populations to construct mixed pools, whereas BSA can use pooled samples from any population, including natural ones and mixtures of any populations. With the reduction of sequencing cost and the development of statistical methods, whole-genome sequencing of pooled individuals has significantly improved the power of BSA, and it has been successfully used in natural population-based mapping (Bastide et al. 2013; Schlötterer et al. 2014).

Genomic selection (GS) or genome-wide selection uses genomewide polymorphic molecular markers to predict complex quantitative traits (Meuwissen et al. 2001). Instead of previous strategies, such as marker-assisted recurrent selection, that need to identify markers of significant effects, GS uses all markers and focuses on an efficient estimation of breeding values, capturing the variation caused by small effect QTL. GS is based on the same hypothesis as GWAS that all of the QTL are in LD with at least one marker (Goddard and Hayes 2007). The effectiveness of GS to predict complex traits has been proven (Rutkoski et al. 2014; Zhang et al. 2015; Zhao et al. 2012). Combined GS with linkage and association mapping has improved breeding efficiency (Cao et al. 2017).

In the present study, 509 maize inbred lines with diverse genetic backgrounds were used in GWAS, BSA, and genomic prediction for identifying resistant QTL for FER in maize. Each inbred line was first genotyped with high-density SNP markers and phenotyped across four environments; then, the materials with extreme phenotypes were selected according to the phenotypic data to form two mixed pools for resequencing. We combined GWAS with BSA to identify associated SNP markers and candidate genes, and finally, genomic prediction was conducted to test the associated markers.

\section{Materials and Methods}

Plant materials, experimental design, and phenotyping. A total of 509 maize inbred lines (423 temperate and 86 tropical/subtropical) were used in the study, including 329 Chinese and 94 U.S. temperate inbreds and 86 tropical/subtropical inbreds from the International Maize and Wheat Improvement Center (CIMMTY). Several key inbred lines that have been widely used in China as parental lines for breeding (Huangzao4, Mo17, Zong31, B73, PHW86, Ye478, and Qi319) were included. Two elite inbred lines, Shen137 (temperate) and CML130 (tropical), were used as checks. Shen137 was extremely resistant to maize FER as identified by multiple years of artificial inoculation (Duan et al. 2015). CML130 from the CIMMYT has moderate resistance (CIMMYT 1998). Detailed information about the plant materials is provided in Supplementary Table S1.

Phenotyping of FER was carried out across four trials in two experimental stations of the Institute of Crop Sciences, Chinese Academy of Agricultural Sciences (Shunyi, Beijing, and Xinxiang, Henan) for 2 years (2017 and 2018). An $\alpha(0,1)$ lattice design with two replicates was utilized. Experimental units were single 4-m row plots each with 17 plants, with $25 \mathrm{~cm}$ between plants and $75 \mathrm{~cm}$ between rows. The planting dates in Shunyi, Beijing, and Xinxiang, Henan, were 2 May and 14 June 2017 and 4 May and 17 June
2018, respectively. The experiments in four environments all utilized sprinkler irrigation and normal agronomic practices. Weather conditions, including rain (millimeters), sun hours, wind speed, relatively humidity, average temperature, and maximum and minimum temperatures (degrees Celsius), were recorded daily during the crop seasons in each location using Vantage Pro 2 Weather Stations (Davis Instruments). Days from sowing to when $50 \%$ of the plants in a row tasseled, silked, and pollinated were recorded as days to tassel (DTT), days to silk (DTS), and days to anthesis (DTA), respectively.

The nail punch/sponge inoculation method was used because it provides the highest level of infection where the inoculum is placed in the side of the ear at 7 days after female flowering (Drepper and Renfro 1990). In this study, the nail punch/sponge and injection inoculation methods were combined. The nail was placed in a piece of broom handle extending $2.3-\mathrm{cm}$ long and 3 to $4 \mathrm{~mm}$ in diameter. FER inoculation with aggressive isolates of $F$. verticillioides was done 7 to 10 days after pollination. The nail was first inserted into the center of the ear through the husk sideways, penetrating the kernels to a depth of about $5 \mathrm{~mm}$, and then, $4 \mathrm{ml}$ of spore suspension with 500,000 spores per milliliter was injected. Because of the different maize flowering dates, inoculation was conducted several times. The inoculation was conducted during 0500 to 0800 or 1500 to $1800 \mathrm{~h}$ to avoid high temperature and strong sunshine as well as to improve the success rate of inoculation. To avoid a nested effect of the genotype within the inoculation, the inoculated lines were bagged. At least 10 maize plants were inoculated per row. At maturity, inoculated ears were harvested by hand, and the FER severity was recorded for each ear as nine grades: 1 (FER score) $=0 \%, 2=$ $1 \%, 3=2$ to $5 \%, 4=6$ to $10 \%, 5=11$ to $20 \%, 6=21$ to $40 \%, 7=$ 41 to $60 \%, 8=61$ to $80 \%$, and $9=81$ to $100 \%$ according to the percentage of infected ear area. A weighted ear rot average was calculated to represent the FER severity.

Genotyping. For DNA extraction, 15 seeds from each inbred line were planted in a seedling tray and grown to the three-leaf stage; then, they were harvested in bulk for DNA extraction. The genomic DNA was extracted by the high-throughput CTAB method (CAASCIMMYT Maize Molecular Breeding Laboratory 2015), and the quality and concentration of DNA were determined by agarose gel electrophoresis and nanodrop. The qualified DNA was genotyped with 55K chip, which contained 55,230 SNP markers (Xu et al. 2017), using the Affymetrix Axiom platform at CapitalBio Technology Corporation. A total of 37,801 SNPs with minor allele frequency $>5.0 \%$ and missing rate $<10.0 \%$ (heterozygous SNPs were scored as missing) were selected and used for GWAS and genomic prediction.

Statistical analyses. Descriptive statistics (mean, range, standard deviation, and coefficient of variation) for phenotypic data were analyzed in Excel 2016. Statistical analyses were performed in Statistical Analysis System (SAS) software version 9.4. Normality of the frequency distributions for FER severity was tested by the Kolmogorov-Smirnov test using the SAS Univariate PROC procedures. Pearson correlation coefficients between FER score and FER severity were computed using the SAS CORR PROC procedures. Analyses of variance (ANOVAs) in different environments were performed using SAS GLM PROC procedures. The single environment heritability $\left(H^{2}\right)$ was estimated as:

$$
H^{2}=\sigma_{G}^{2} /\left(\sigma_{G}^{2}+\sigma_{\varepsilon}^{2} / r\right)
$$

where $\sigma_{G}^{2}$ represents the genetic variance, $\sigma_{\varepsilon}^{2}$ is the residual error variance, and $r$ is the number of replications in each environment. Broad-sense heritability $\left(H^{2}\right)$ was estimated as:

$$
H^{2}=\sigma_{G}^{2} /\left(\sigma_{G}^{2}+\sigma_{G E}^{2} / E+\sigma_{\varepsilon}^{2} / l r\right)
$$

where $\sigma_{G E}^{2}$ is genotype $\times$ environment interaction variance and $l$ is the number of environments.

Best linear unbiased estimators (BLUEs) of the FER severity in the single environment and multiple environments were evaluated in $\mathrm{R}$ package "Ime4" (R Core Team 2015). For the single environment, we fitted a mixed linear model with genotypes as fixed effects and replication within environment as random effects. For the multiple 
environments, we fitted a mixed linear model with genotypes as fixed effects and environment, genotype $\times$ environment, and replication within environment as random effects using the following mathematical model:

$$
y_{i l j}=\mu+g_{i}+e_{l}+g e_{i l}+b_{j}+\varepsilon_{i l j}
$$

where $y_{i l j}$ represents the phenotypic value, $\mu$ is the grand mean value in all environments, $g_{i}$ is the effect of the $i$ th genotype, $e_{l}$ is the effect of the $l$ th environment, $g e_{i l}$ is the effect of the genotype $\times$ environment interaction, $b_{j}$ is the effect of $j$ th replication, and $\varepsilon_{i l j}$ is the model residual. The phenotypic and genotypic Pearson correlation coefficients between environments were computed based on the mean values and BLUE values, respectively, using the SAS CORR PROC procedures. The Pearson correlation coefficient is defined by:

$$
r_{x y}=\frac{\sum\left(x_{i}-\bar{x}\right) \sum\left(y_{i}-\bar{y}\right)}{\sqrt{\left(x_{i}-\bar{x}\right)^{2}} \sqrt{\left(y_{i}-\bar{y}\right)^{2}}}
$$

where $\bar{x}=\frac{1}{n} \sum_{i=1}^{N} x_{i}$ denotes the mean of $x . \bar{y}=\frac{1}{n} \sum_{i=1}^{N} y_{i}$ denotes the mean of $y$. One-way ANOVA in R package (R Core Team 2015) was performed to reveal the difference of FER resistance among eight major maize germplasm groups (SPT, LRC, PA, PB, Reid, Lancaster, Iodent, and subtropical/tropical) (Supplementary Table S1).

LD analysis and GWAS. TASSEL 5.0 was used to calculate LD $\left(r^{2}\right)$ between pairwise SNPs for the association panel. The average distance of LD decay across the entire genome was estimated using all LD data in SAS Nlin PROC procedures. To observe the trend of LD decay, 2,000 pairwise $\mathrm{LD} r^{2}$ estimates randomly selected from 10 chromosomes were plotted versus the corresponding pairwise genetic distances. The LD attenuation distance around each SNP was used to search for candidate genes that may lead to any significant association in GWAS. GWAS was carried out by mixed linear model in TASSEL 5.0 software by accounting for the principal component analysis and kinship matrix. The BLUE values for each inbred line in the single environment and multiple environments were used as the phenotypes for association mapping. The mixed linear model can be described as follows:

$$
Y=X \beta+Z u+\varepsilon
$$

where $Y$ is the observed value vector, $\beta$ is the fixed effect vector that includes genetic markers and population structure factors, $u$ is the random effect vector, $X$ and $Z$ are the known design matrices, and $\varepsilon$ is the random residual effect vector.

Bonferroni test (0.05/number of hypotheses tested) was used to select the SNPs significantly associated with FER; however, only one SNP was identified in Shunyi 2017, or two SNPs were identified using BLUE values. Therefore, a moderated stringency threshold of $-\log (1 /$ number of hypotheses tested $)=P<2.65 \times 10^{-5}$ was chosen to determine significantly associated SNPs based on the suggestion of Han et al. (2009) and Yang et al. (2011). Considering that the FER was determined by many genes, using a less stringent threshold of $1.0 \times 10^{-4}$ in this study is reasonable. All of the candidate genes were identified according to the information available in the MaizeGDB database (https://www.maizegdb.org/gbrowse). The genes containing or adjacent to the SNPs significantly associated with FER resistance were identified using the predicted genome screened from the annotated B73 reference genome.

BSA. According to the BLUE value in the multiple environments, 30 resistant and 30 susceptible inbred lines were selected. DNA was extracted individually by the high-throughput CTAB method, and an equal amount of the extraction from each individual was mixed to form resistant and susceptible DNA pools, which were sent to Berry Genomics Corporation for resequencing (four libraries built for each pool, Illumina Hiseq2500 sequencer, insertion fragment $250 \mathrm{bp}$, and double-ended sequencing $150 \mathrm{bp}$ ). For data analysis, Fastx-toolkit (v0.0.14-1) was use to filter out the low-quality reads (Li and Durbin 2009). Burrows-Wheeler Aligner (v0.7.5a-r405) was used to align the clean reads to the reference maize genome (Zea_mays AGPv3.27). The alignment results were saved in binary alignment map (BAM; Sequence Alignment/Map [SAM]) format files. Sequence Alignment/Map Tools (SAMtools; v0.1.19) were applied to sort and transfer the SAM to BAM format files ( $\mathrm{Li}$ et al. 2009). Picard tools (v1.57) were used to excluded the duplicates, and the final sorted BAM format files were saved for the downstream analysis. Based on the alignment result, GATK (v3.8) and SAMtools were used for SNP calling (McKenna et al. 2010). As a natural population, there are no parents that can be used to filter polymorphic information. To reduce sequencing errors and mapping errors, the filtering criteria for polymorphic loci are as follows: (1) clean $Q>$ 20, (2) nonpolymorphic SNPs excluded, and (3) frequency of the $\mathrm{SNP}$ in two mixed pools $<0.3$.

The Euclidean distance (ED) algorithm was used to estimate the difference between the two mixed DNA pools using the following formula:

$$
E D=\sqrt{(A T-A S)^{2}+(C T-C S)^{2}+(G T-G S)^{2}+(T T-T S)^{2}}
$$

where $A T, C T, G T$, and $T T$ were used to represent frequencies of $\mathrm{A}$, $\mathrm{C}, \mathrm{G}$, and $\mathrm{T}$ bases in the resistant pool, respectively, whereas $A S, C S$, $G S$, and $T S$ were used to represent the frequencies of $\mathrm{A}, \mathrm{C}, \mathrm{G}$, and $\mathrm{T}$ bases in the susceptible pool, respectively. The ED value represents the size of the difference between the two DNA pools.

Genome prediction by cross-validation. To validate the result of GWAS for breeding, the markers randomly selected and the markers associated with FER severity as revealed by GWAS were used for genomic prediction using five classical models, including genomic best linear unbiased prediction (GBLUP), three Bayesian models (BayesA, BayesB, and BayesC) in BGLR packages, and ridge regression-best linear unbiased prediction (rrBLUP) in rrBLUP packages (R Core Team 2015). The markers associated with FER severity were selected by the $\log _{10}(P)$ values from large to small according to the results of GWAS.

Prediction accuracy $\left(r_{\mathrm{MG}}\right)$ was evaluated through a fivefold crossvalidation $(80 \%$ of the inbred lines in the population were randomly selected as the training population, and the remaining $20 \%$ were selected as the validation population). To estimate the effects of marker density and training population size on prediction accuracy, different marker numbers $(50,100,200,500,1,000,2,000,4,000$,

\begin{tabular}{|c|c|c|c|c|c|c|c|c|}
\hline Enva & $N$ & Mean (range; \%) & $\mathbf{S D}^{\mathbf{b}}$ & Skewness & Kurtosis & $\sigma_{G}^{2}$ & $\sigma_{G E}^{2}$ & $\boldsymbol{H}^{2}$ \\
\hline Shunyi 2017 & 376 & $20.36(1.78-86.67)$ & 0.14 & 1.378 & 2.265 & $0.0113^{\mathrm{c}}$ & & 0.68 \\
\hline Xinxiang 2017 & 442 & $21.13(1.25-100)$ & 0.19 & 1.510 & 2.276 & $0.0222^{c}$ & & 0.69 \\
\hline Shunyi 2018 & 412 & $22.24(0-100)$ & 0.20 & 1.296 & 1.232 & $0.0313^{\mathrm{c}}$ & & 0.65 \\
\hline Xinxiang 2018 & 435 & 23.07 (0-91.80) & 0.23 & 1.144 & 0.365 & $0.0448^{\mathrm{c}}$ & & 0.76 \\
\hline Combined & 492 & $21.77(0.33-84.59)$ & 0.16 & 1.211 & 1.398 & $0.0193^{\mathrm{c}}$ & $0.0086^{\mathrm{c}}$ & 0.78 \\
\hline
\end{tabular}
$8,000,10,000,20,000$, and all markers) and training population sizes

Table 1. Descriptive statistics, genetic variance $\left(\sigma_{G}^{2}\right)$, genotype-environment interactions variance $\left(\sigma_{G E}^{2}\right)$, and broad-sense heritability $\left(H^{2}\right)$ for Fusarium ear rot resistance in the association panel

a Env, environment.

b SD, standard deviation.

c Significant at $P=0.01$. 
(ranging from 10 to $90 \%$ of the total population size) were used for testing. Prediction accuracy was estimated according to Pearson correlation coefficients between the observed phenotypic values and the predicted breeding values averaged over all 100 replications. GBLUP model and 4,000 trait-related markers were used to predict breeding values of the 30 most resistant lines, which were compared with the observed phenotypic values based on Spearman correlation coefficient.

\section{Results}

Phenotypic variation, high heritability estimates, and donor lines for FER resistance. Significant $(P<0.01)$ genotypic variation $\left(\sigma_{G}^{2}\right)$ for ear rot resistance was observed across the four environments (Table 1), and maize inbred lines showed 1 to 9 of FER severity scales (Fig. 1). FER score and FER severity were positively correlated $(r=0.9583$ to $0.9720, P<0.001$ ) (Supplementary Figs. S1 and S2). Therefore, subsequent data and GWAS analysis were performed using FER severity only. The means of FER severity in four

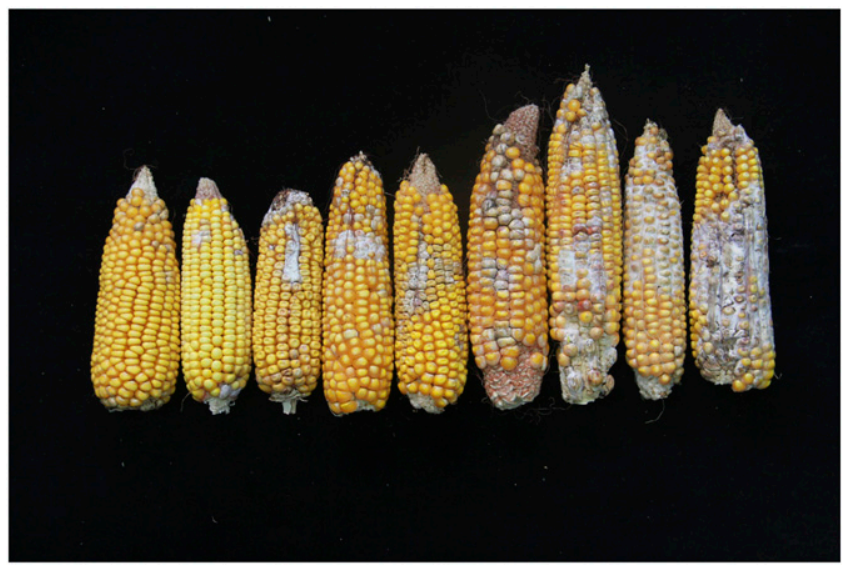

Fig. 1. Phenotypic variation in Fusarium ear rot severity at harvest among the 509 diverse inbred lines. Left to right images represent the 1 to 9 scale, respectively. environments (Shunyi 2017, Xinxiang 2017, Shunyi 2018, and Xinxiang 2018) were $20.36 \%$ (1.78 to $86.67 \%$ ), $21.13 \%$ (1.25 to $100.00 \%$ ), $22.24 \%$ ( 0 to $100.00 \%$ ), and $23.07 \%$ (0 to $91.80 \%$ ), respectively (Table 1$)$, showing significant $(P<0.01)$ difference among environments $\left(\sigma_{E}^{2}\right)$. In the combined analysis, mean FER severity across four environments ranged from 0.33 to $84.59 \%$, with an overall mean of $21.77 \%$ (Table 1). Significant genotypic components of variance $\left(\sigma_{G}^{2}\right)$ and genotype $\times$ environment interaction $\left(\sigma_{G E}^{2} ; P<0.01\right)$ were detected by combined ANOVA analysis. The Kolmogorov-Smirnov test showed that FER severity was not normally distributed $(P<0.01)$, with a skew toward the lower level of infection owing to the higher frequencies of scales 4 and 5 (corresponding to FER severity 6 to $10 \%$ and 11 to $20 \%$, respectively) (Fig. 2). Reflectance kurtosis analysis showed that the maize FER resistance was continuously distributed as a quantitative trait (Fig. 2 and Table 1).

In the same location, the 2 years (2017 and 2018) had very similar weekly sun hours, humidity, average temperature, and maximum and minimum temperatures, but they had unevenly distributed rainfall (Supplementary Table S2). The estimated heritability $\left(\mathrm{H}^{2}\right)$ of the FER severity ranged from 0.65 in Shunyi 2018 to 0.76 in Xinxiang 2018, and the heritability of line means across four environments was 0.78 , greater than that for any single environment. High heritability estimates indicate that FER resistance is largely controlled by

Table 2. Phenotypic correlation coefficient (above the diagonal) and genetic correlation coefficient (below the diagonal) in four environments

\begin{tabular}{lcccc}
\hline Env $^{\mathrm{a}}$ & $\begin{array}{c}\text { Shunyi } \\
\mathbf{2 0 1 7}\end{array}$ & $\begin{array}{c}\text { Xinxiang } \\
\mathbf{2 0 1 7}\end{array}$ & $\begin{array}{c}\text { Shunyi } \\
\mathbf{2 0 1 8}\end{array}$ & $\begin{array}{c}\text { Xinxiang } \\
\mathbf{2 0 1 8}\end{array}$ \\
\hline Shunyi 2017 & $1.0000^{\mathrm{b}}$ & $0.8576^{\mathrm{b}}$ & $0.3855^{\mathrm{b}}$ & $0.4944^{\mathrm{b}}$ \\
$\begin{array}{l}\text { Xinxiang } \\
\text { 2017 }\end{array}$ & $0.8365^{\mathrm{b}}$ & $1.0000^{\mathrm{b}}$ & $0.3786^{\mathrm{b}}$ & $0.4911^{\mathrm{b}}$ \\
$\begin{array}{l}\text { Shunyi 2018 } \\
\text { Xinxiang }\end{array}$ & $0.3863^{\mathrm{b}}$ & $0.3938^{\mathrm{b}}$ & $1.0000^{\mathrm{b}}$ & $0.4091^{\mathrm{b}}$ \\
2018 & $0.5201^{\mathrm{b}}$ & $0.5198^{\mathrm{b}}$ & $0.4340^{\mathrm{b}}$ & $1.0000^{\mathrm{b}}$ \\
\hline
\end{tabular}

a Env, environment.

${ }^{\text {b }}$ Significant at $P=0.01$.
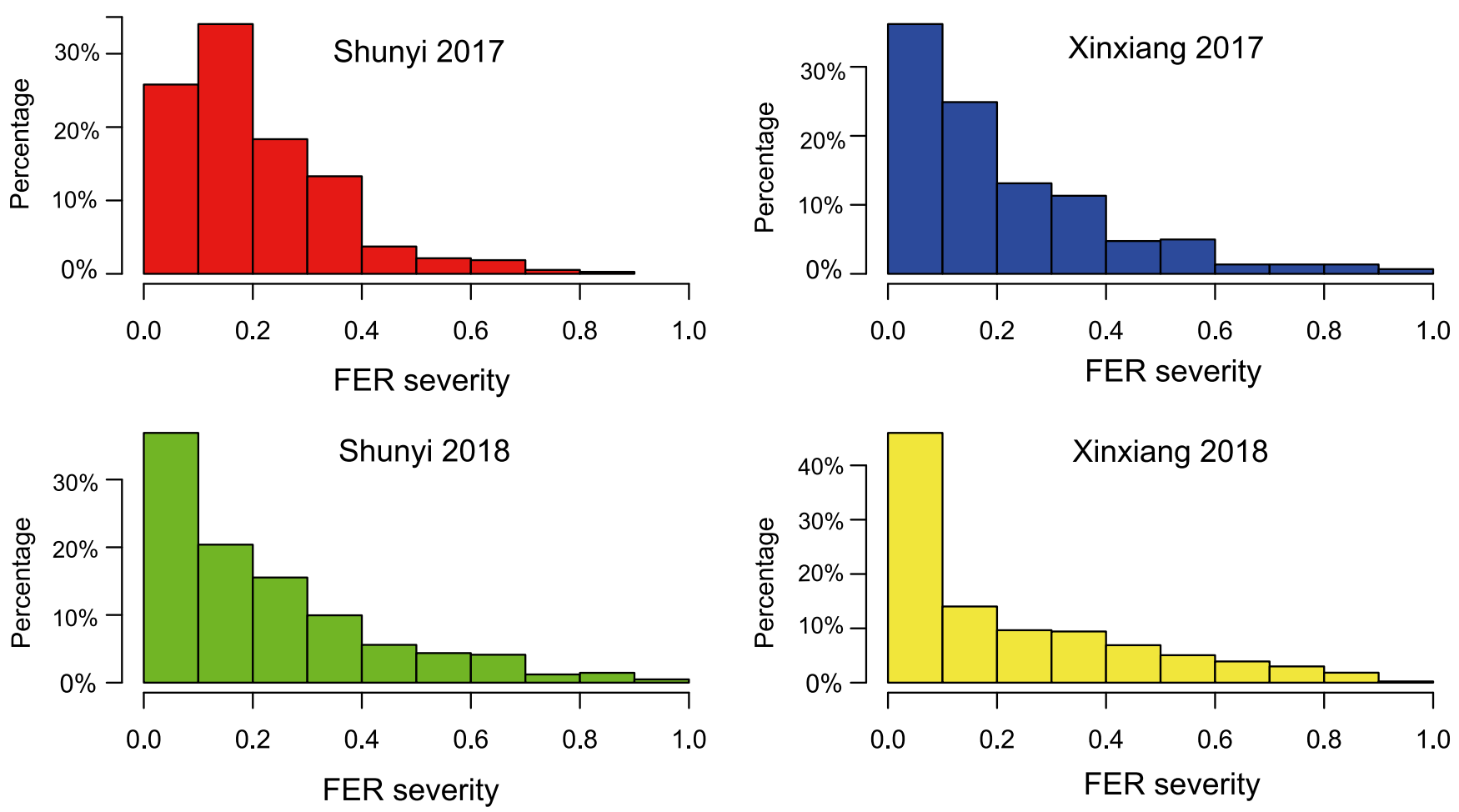

Fig. 2. Distribution of Fusarium ear rot (FER) severity of the maize association panel in Shunyi 2017, Xinxiang 2017, Shunyi 2018, and Xinxiang 2018. 
genetic factors. The phenotypic correlation $(r=0.3786$ to 0.8576$)$ and the genetic correlation ( $r=0.3863$ to 0.8365$)$ in four environments were highly significant $(P<0.01)$ (Table 2$)$. A much stronger genotypic correlation was observed between Shunyi 2017 and Xinxiang 2017 than any other pairwise environments. Some inbred lines had very low FER severity $(<0.05$ in each of the four environments). Thirty inbred lines were identified as FER resistant by combined analysis of phenotypic data across the four environments (Supplementary Table S3). These inbred lines are potential germplasm sources for resistance to FER.

Table 3. Single-nucleotide polymorphism (SNP) and most likely candidate genes associated with Fusarium ear rot resistance

\begin{tabular}{|c|c|c|c|c|c|c|}
\hline SNP & Bin & Position $^{\mathbf{a}}$ & $P$ value & $R^{2}$ & Candidate & Annotation \\
\hline AX-91451743 & 1.02 & $22,891,134$ & 8.34E-05 & 0.0370 & GRMZM5G863656 & Ribosomal S3Ae family \\
\hline AX-90614231 & 1.04 & $68,117,872$ & $2.24 \mathrm{E}-05$ & 0.0400 & GRMZM2G305400 & Cyclin-D2-1 \\
\hline AX-90599863 & 1.07 & $226,233,417$ & $1.04 \mathrm{E}-07$ & 0.0640 & GRMZM5G869779 & Synthase isoform $\mathrm{X} 1$ \\
\hline AX-86307842 & 1.08 & $229,134,311$ & $1.25 \mathrm{E}-05$ & 0.0430 & GRMZM2G071304 & DNA ligase \\
\hline AX-123944580 & 2.02 & $4,589,840$ & $3.51 \mathrm{E}-06$ & 0.0487 & GRMZM2G386362 & Scarecrow-like protein 6 \\
\hline AX-86256935 & 2.03 & $16,851,480$ & $1.85 \mathrm{E}-05$ & 0.0446 & GRMZM2G096086 & Sytochrome P450 87A3 isoform X1 \\
\hline AX-86323066 & 2.03 & $17,278,628$ & $5.10 \mathrm{E}-05$ & 0.0367 & GRMZM2G318956 & Spc97/Spc98 family \\
\hline AX-91836764 & 2.08 & $215,048,177$ & $3.69 \mathrm{E}-06$ & 0.0479 & GRMZM5G810312 & RNA-dependent RNA polymerase \\
\hline AX-90575891 & 3.08 & $213,419,219$ & 4.75E-05 & 0.0369 & GRMZM2G033515 & Seed maturation protein PM41 \\
\hline AX-91411838 & 4.04 & $29,963,655$ & $5.16 \mathrm{E}-05$ & 0.0365 & GRMZM2G309109 & MRE11B \\
\hline AX-123947333 & 4.04 & $29,964,406$ & $3.55 \mathrm{E}-06$ & 0.0483 & GRMZM2G309109 & MRE11B \\
\hline AX-123944911 & 4.04 & $29,965,113$ & $5.35 \mathrm{E}-05$ & 0.0364 & GRMZM2G309109 & MRE11B \\
\hline AX-90915634 & 4.09 & $225,260,406$ & $9.51 \mathrm{E}-05$ & 0.0340 & GRMZM2G085751 & bhlh24: bHLH-transcription factor 24 \\
\hline AX-91654018 & 5.03 & $65,968,046$ & $9.56 \mathrm{E}-05$ & 0.0348 & GRMZM2G033829 & Protein disulfide isomerase4 precursor \\
\hline AX-123947982 & 5.04 & $168,494,274$ & 8.99E-05 & 0.0343 & GRMZM2G032628 & Amylose extender1 \\
\hline AX-86249772 & 6.01 & $7,783,749$ & $1.29 \mathrm{E}-05$ & 0.0434 & GRMZM2G058787 & - \\
\hline AX-86316371 & 6.01 & $8,683,611$ & $9.96 \mathrm{E}-05$ & 0.0337 & GRMZM2G060106 & Hypothetical protein \\
\hline AX-91074043 & 8.02 & $20,094,843$ & $7.41 \mathrm{E}-05$ & 0.0351 & GRMZM2G342647 & nactf52: NAC-transcription factor 52 \\
\hline AX-91154120 & 9.06 & $146,327,273$ & $9.64 \mathrm{E}-06$ & 0.0445 & GRMZM2G046601 & gln3: glutamine synthetase3 \\
\hline AX-91805352 & 9.06 & $146,327,288$ & $2.13 \mathrm{E}-06$ & 0.0653 & GRMZM2G046601 & gln3: glutamine synthetase3 \\
\hline AX-86255689 & 10.03 & $14,501,044$ & $3.79 \mathrm{E}-08$ & 0.0685 & GRMZM2G083347 & NAC transcription factor NAM-1 \\
\hline AX-91183581 & 10.04 & $100,871,892$ & $6.13 \mathrm{E}-06$ & 0.0494 & GRMZM2G004211 & APx3: Peroxisomal Ascorbate Peroxidase \\
\hline AX-91832354 & 10.05 & $136,646,197$ & $4.41 \mathrm{E}-05$ & 0.0414 & GRMZM2G006212 & MATE efflux family protein 5 like \\
\hline
\end{tabular}

a The physical position based on B73 reference genome v3(B73 RefGen_v3).

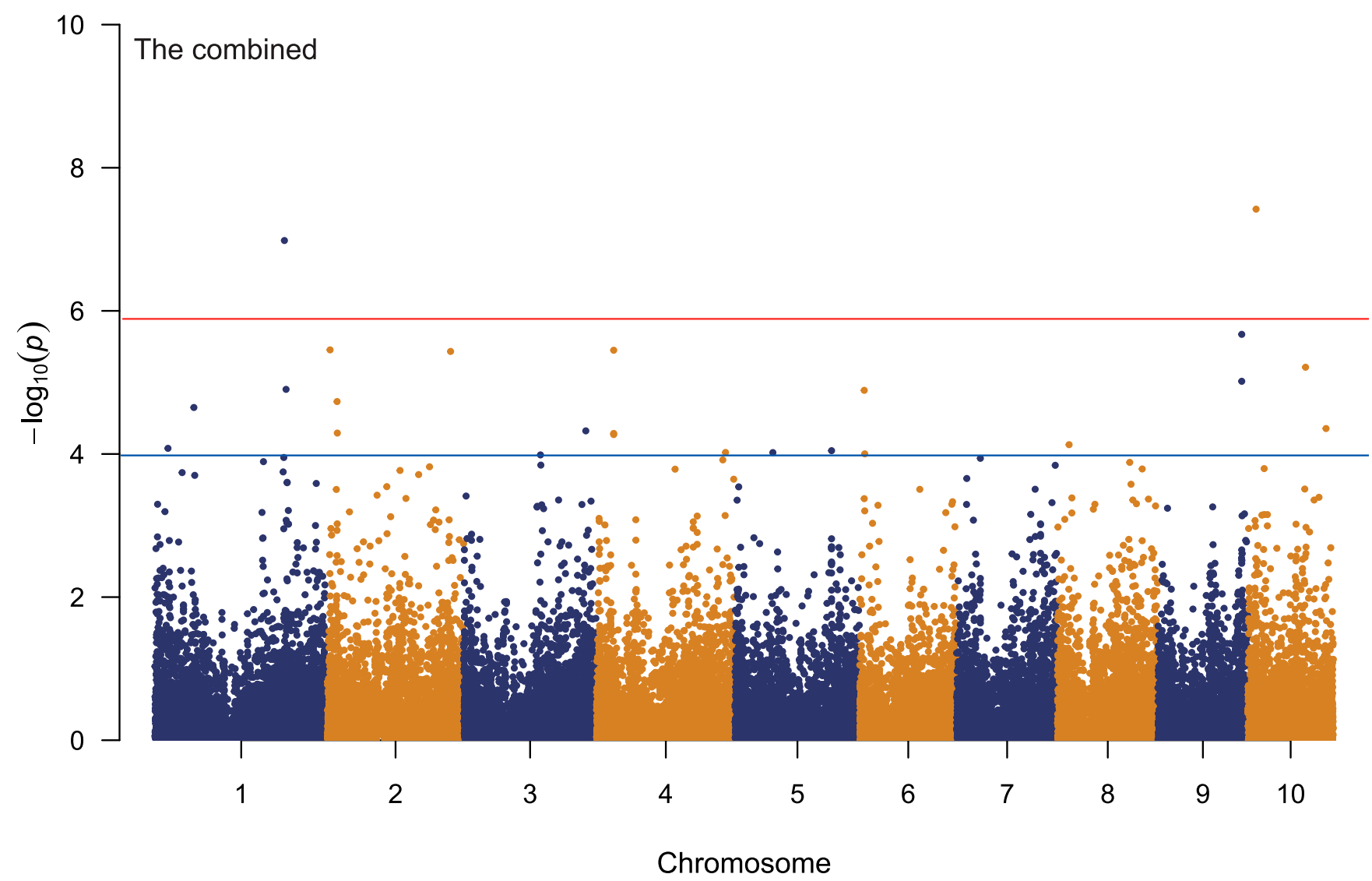

Fig. 3. Genome-wide association study results for Fusarium ear rot severity in the maize association panel and Manhattan plots for the combined. The blue line shows genome-wide significance with a low stringent threshold of $1.0 \times 10^{-4}$. The red line shows genome-wide significance with a more stringent threshold of $1.32 \times 10^{-6}[-\log (0.05 / 37,801)]$. 
Genetic complexity for FER resistance revealed by GWAS. Integrated with the population structure and familial relatedness, a mixed linear model was fitted to scan 37,801 SNPs across the whole genome. Quantile-quantile plots implied that the population structure and familial relatedness were well controlled in the GWAS (Supplementary Fig. S3). With a low stringent threshold of $1.0 \times 10^{-4}, 13,5$, 9, and 7 SNPs, which were distributed on all chromosomes, were found to be associated with FER in four environments: Shunyi 2017, Xinxiang 2017, Shunyi 2018, and Xinxiang 2018, respectively (Supplementary Fig. S4). The 34 SNPs identified each accounted for 3.3 to $6.7 \%$ of the phenotypic variance (Table 3 ). With a moderately stringent threshold of $2.65 \times 10^{-5}[-\log (1 / 37,801)]$, only 10 SNPs were identified to be associated with FER, with no associated SNPs found in Xinxiang 2017. At a more stringent threshold of $1.32 \times 10^{-6}$ $[-\log (0.05 / 37,801)]$ by Bonferroni test, only one SNP (3_219011459 on chromosome 3) was identified in Shunyi 2017. Although no identified SNPs were shared across two environments, significant correlation $(r=0.0091$ to $r=0.1823 ; P<0.05)$ of $-\log _{10}(P)$ for all 37,801 SNPs between pairwise environments indicates that GWAS of FER resistance is reliable (Supplementary Table S4). The correlation between Shunyi 2017 and Xinxiang 2017 was stronger than any other pairwise environments, which is in line with the phenotypic and genetic correlation.

In the combined analysis using BLUE values from the four environments, 23 SNPs were identified to be associated with FER resistance at a low stringent threshold of $1.0 \times 10^{-4}$ (Fig. 3). At a moderately stringent threshold of $2.65 \times 10^{-5}$ [- $\left.\log (1 / 37,801)\right], 12$ SNPs were identified. At a more stringent threshold of $1.32 \times 10^{-6}$ $[-\log (0.05 / 37,801)]$, however, only two SNPs (1_226233417 on chromosome 1 and 10_14501044 on chromosome 10) could be identified. These two SNPs were also detectable at 0.01 significance level (threshold of $2.65 \times 10^{-7}[-\log (0.01 / 37,801)]$ ) (Fig. 3 and Table $3)$. No SNPs were overlapped with those identified by singleenvironment analysis. These results indicate that FER resistance is multigenic and genetically complicated. Majority of the QTL for FER resistance identified by the 23 SNPs were reported previously. For example, the locus on bin 2.03 has been reported by two QTL mapping studies (Giomi et al. 2016; Maschietto et al. 2017) and one association mapping (Chen et al. 2016). The locus on bin 5.04 has also been reported in two QTL mappings (Chen et al. 2012; Ding et al. 2008) and one association mapping (Chen et al. 2016). The loci on bins 2.02, 3.05, and 7.05 have been reported by Coan et al. (2018), and the loci on bins 1.04, 1.07, 2.03, 3.05, 4.04, 4.09, 5.03, and 9.03 have been reported by Chen et al. (2016).

Associated SNPs identified within important chromosome bins. Using the bulked samples with extreme phenotypes (Supplementary Fig. S5 and Supplementary Table S3), the susceptible pool produced $572,478,839$ clean reads $(\mathrm{Q} 30=94.4 \%)$ and $171,743,651,700$ clean bases $(\mathrm{Q} 30=92.9 \%)$, whereas the resistant pool produced $592,184,520$ clean reads $(\mathrm{Q} 30=94.2 \%)$ and $177,655,356,000$ clean bases $(\mathrm{Q} 30=92.4 \%)$. By comparing the clean reads with the reference genome, 98.9 and $98.5 \%$ clean reads from the susceptible and resistant pools were mapped to the reference genome, with the duplicate rates of 13.29 and $13.90 \%$, respectively. A total of $34,787,877$ SNPs were detected, of which $2.6 \%$ were homozygous, whereas $97.4 \%$ were heterozygous. A total of $2,061,974$ indels were detected, of which $18.4 \%$ were homozygous, whereas $81.7 \%$ were heterozygous. After filtering polymorphic information, $14,314,114$ SNPs and 1,211,732 indels were identified between the two pools.

The average of ED in the 1-Mb window was selected to represent the distribution of ED. Three confidence levels of 99, 95, and $90 \%$ were selected as thresholds of correlation (Fig. 4). By integrating ED of SNPs and indels, at the $90 \%$ confidence level, significant correlation intervals were identified on chromosome bins 3.04, 3.05, $3.06,4.05,7.05,9.03,10.03$, and 10.04 (Table 4 ), but at the $95 \%$ confidence level, significant correlation intervals were only identified on chromosome bins 3.04 and 10.03 (Table 4 and Supplementary Figs.
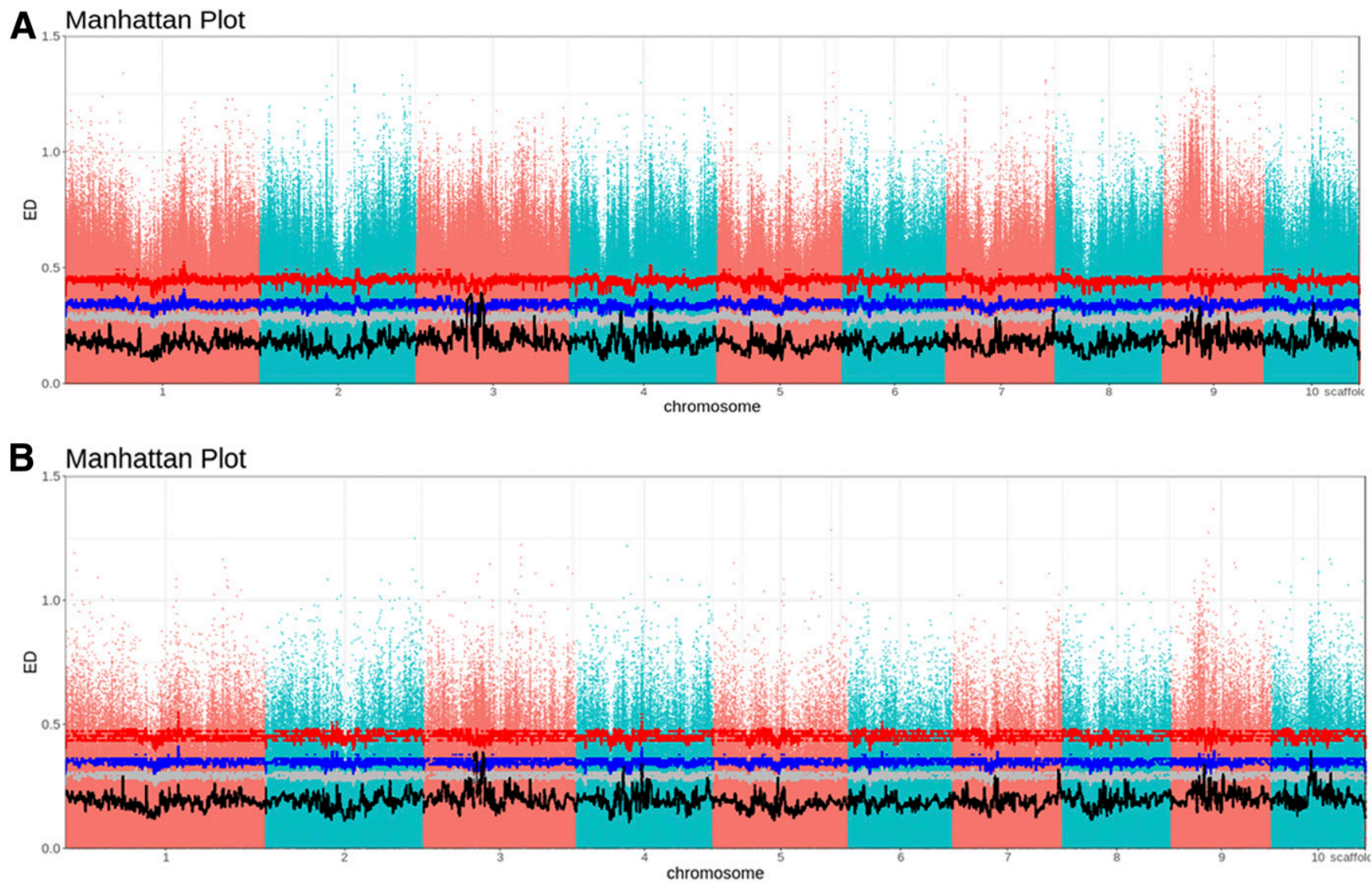

Fig. 4. The distribution of Euclidean distance (ED)-associated values of A, single-nucleotide polymorphism and $B$, indel on chromosome. The ED value represents the size of the difference between the two DNA pools. The red lines, blue lines, and gray lines represent 99,95 , and $90 \%$ confidence levels, respectively. 
S6 and S7). No correlation intervals were identified at the $99 \%$ confidence level. These loci have been reported previously by linkage or association mapping, except chromosome bin 10.04. The locus on chromosome bin 3.05 has been reported by two QTL mappings (Giomi et al. 2016; Maschietto et al. 2017) and one association mapping (Chen et al. 2016), whereas bin 10.03 has been reported by one QTL mapping (Ding et al. 2008) and two association mappings (Chen et al. 2016; Coan et al. 2018). The loci on chromosome bins $3.04,3.06,4.05$, and 9.03 have been reported by at least two different studies (Chen et al. 2012, 2016; Coan et al. 2018; Ding et al. 2008; Robertson-Hoyt et al. 2006; Zila et al. 2014). The locus on chromosome bin 7.05 has been reported in the work by Coan et al. (2018).

Candidate genes associated with FER resistance. LD decay declined rapidly between 0 and $100 \mathrm{~kb}$ and then, slowly (Supplementary Fig. S8). Using the model of

$$
y=a+b \times \log (x)
$$

the average distance of LD decay across the entire genome was $219 \mathrm{~kb}$ when $r^{2}=0.1$, and this is line with our previous report (Xu et al. 2017). A total of 23 different SNPs identified by GWAS using BLUE values across the four environments were adjacent to or in 23 candidate genes (Table 3), including transcription factors, enzymes, protein metabolism, hypothetical protein, and other biological processes. The most significant 10_14501044 on chromosome 10 contained the gene GRMZM2G083347, a transcription factor. The significant 1_226233417 on chromosome 1 is adjacent to the gene GRMZM5G869779, which encodes synthase isoform X1. These results reveal the complex nature of FER resistance and various mechanisms that might be involved in conditioning FER resistance, including complex biosynthesis processes and interactions between multiple metabolic pathways. By BSA, 87 candidate genes were identified on chromosomes $3,4,7,9$, and 10 within the $90 \%$ confidence interval (Table 4). Although the loci on chromosome bins
10.03 and 10.04 were identified by both GWAS and BSA, no candidate genes were found by both methods.

Genomic prediction accuracy by trait-related markers. No significant differences in prediction accuracy were observed among the five GS models (BayesA, BayesB, BayesC, GBLUP and rrBLUP); values were $0.355,0.338,0.357,0.367$, and 0.351 , respectively (Fig. 5A). The effects of randomly selected markers and traitrelated markers on prediction accuracy were evaluated using the GBLUP model. Significantly different prediction accuracies $(P<$ 0.01 ) between four environments were found (Fig. 5B), and the highest prediction accuracy occurred in Xinxiang 2018. The prediction accuracy continuously increased from 0.172 to 0.374 as the training population size increased (Fig. 5C).

Under different levels of marker density, prediction accuracy increased from 0.214 (50 SNPs) to 0.364 (10,000 SNPs) for randomly selected markers and from 0.291 (50 SNPs) to 0.426 (10,000 SNPs) for trait-related markers (Fig. 5D). Approximately 4,000 SNP markers were enough for the natural population to achieve good prediction accuracy. Under different levels of marker density, traitrelated markers always provided higher prediction accuracy than randomly selected markers (Fig. 5D).

The predicted breeding values for the 30 most resistance lines in four environments are provided in Supplementary Table S5, which were significantly correlated $(P<0.05)$ with the observed phenotypic values in Xinxiang $2017(r=0.367)$ and Xinxiang $2018(r=$ 0.441 ) but were not significantly correlated with those in Shunyi $2017(r=0.241)$ or Shunyi $2018(r=0.214)$. The difference in prediction accuracy between randomly selected markers and traitrelated markers decreased as the number of markers increased to a certain level. When marker numbers increased from 50 to 100 , 200 , and 500, the differences in prediction accuracy were 0.076 , $0.070,0.076$, and 0.069 , respectively. When the marker numbers increased to 20,000 and 30,000 , however, the differences in

Table 4. The association region and candidate genes associated with Fusarium ear rot resistance under the $90 \%$ confidence level through bulked sample analysis

\begin{tabular}{|c|c|c|c|c|c|c|c|}
\hline Bin & Start & End & Length (bp) & nSNPs $^{\mathbf{a}}$ & peakED $^{\mathbf{b}}$ & $\operatorname{avgED}{ }^{c}$ & Gene no. \\
\hline \multirow[t]{3}{*}{3.04} & $75,183,257$ & $85,364,753$ & $10,181,496$ & 39,731 & 0.3859 & 0.3651 & 14 \\
\hline & $96,980,041$ & $98,255,386$ & $1,275,345$ & 9,800 & 0.3422 & 0.3173 & 3 \\
\hline & $99,217,104$ & $104,050,694$ & $4,833,590$ & 28,073 & 0.3920 & 0.3487 & 11 \\
\hline \multirow[t]{2}{*}{3.05} & $152,516,144$ & $152,518,669$ & 2,525 & 2 & 0.2781 & 0.2780 & - \\
\hline & $152,602,024$ & $152,645,122$ & 43,098 & 3 & 0.2747 & 0.2740 & 1 \\
\hline 3.06 & $179,976,269$ & $180,226,206$ & 249,937 & 1,683 & 0.2903 & 0.2871 & 3 \\
\hline \multirow[t]{11}{*}{4.05} & $78,798,688$ & $78,845,455$ & 46,767 & 411 & 0.2909 & 0.2888 & 1 \\
\hline & $78,869,710$ & $79,386,254$ & 516,544 & 3,222 & 0.2977 & 0.2941 & 4 \\
\hline & $79,910,299$ & $80,821,998$ & 911,699 & 3,939 & 0.3121 & 0.3021 & 3 \\
\hline & $138,006,890$ & $138,245,669$ & 238,779 & 1,814 & 0.3000 & 0.2934 & 2 \\
\hline & $138,275,254$ & $138,333,224$ & 57,970 & 270 & 0.3037 & 0.3024 & 2 \\
\hline & $138,413,830$ & $138,418,957$ & 5,127 & 35 & 0.3077 & 0.3076 & 1 \\
\hline & $138,627,053$ & $138,985,575$ & 358,522 & 2,474 & 0.3176 & 0.3150 & 2 \\
\hline & $139,051,556$ & $139,100,142$ & 48,586 & 256 & 0.3186 & 0.3184 & 2 \\
\hline & $139,445,110$ & $139,465,677$ & 20,567 & 81 & 0.3220 & 0.3218 & 1 \\
\hline & $139,928,757$ & $140,238,787$ & 310,030 & 1,892 & 0.3333 & 0.3281 & 2 \\
\hline & $140,289,865$ & $141,067,079$ & 777,214 & 4,890 & 0.3195 & 0.3085 & 3 \\
\hline 7.05 & $172,545,046$ & $172,844,001$ & 298,955 & 2,111 & 0.3151 & 0.3077 & 13 \\
\hline \multirow[t]{5}{*}{9.03} & $40,957,036$ & $41,047,114$ & 90,078 & 678 & 0.3094 & 0.3067 & 1 \\
\hline & $41,064,835$ & $41,412,706$ & 347,871 & 2,391 & 0.3128 & 0.3081 & 2 \\
\hline & $49,280,929$ & $50,048,096$ & 767,167 & 6,898 & 0.3323 & 0.3181 & 3 \\
\hline & $67,362,001$ & $67,690,740$ & 328,739 & 52 & 0.2858 & 0.2825 & 2 \\
\hline & $96,275,086$ & $96,333,424$ & 58,338 & 372 & 0.3049 & 0.3043 & 1 \\
\hline \multirow[t]{4}{*}{10.03} & $70,704,281$ & $70,752,958$ & 48,677 & 7 & 0.2898 & 0.2893 & 1 \\
\hline & $74,060,710$ & $75,145,090$ & $1,084,380$ & 5,924 & 0.3451 & 0.3302 & 3 \\
\hline & $76,369,507$ & $77,174,502$ & 804,995 & 444 & 0.3089 & 0.3047 & 1 \\
\hline & $81,430,561$ & $82,065,286$ & 634,725 & 367 & 0.3061 & 0.2993 & 2 \\
\hline \multirow[t]{2}{*}{10.04} & $89,367,648$ & $89,539,656$ & 172,008 & 84 & 0.2777 & 0.2753 & 1 \\
\hline & $112,298,844$ & $112,311,007$ & 12,163 & 117 & 0.2835 & 0.2832 & 2 \\
\hline
\end{tabular}

a nSNP, high-quality single-nucleotide polymorphism/indel number of the quantitative trait loci on the chromosome.

$\mathrm{b}$ peakED, the peak of Euclidean distance corresponding to the quantitative trait loci.

${ }^{c}$ avgED, the average of Euclidean distance corresponding to the quantitative trait loci. 
prediction accuracy reduced to 0.027 and 0.026 , respectively. Therefore, when the number of markers reached 10,000, randomly selected and trait-related markers provided almost the same prediction accuracy.

\section{Discussion}

Maize is one of the major crops that utilizes heterosis. Establishment of heterotic groups and understanding of heterotic patterns are important for hybrid maize breeding. Exploring FER resistance in different maize germplasm or heterotic groups not only can help identify the resistant germplasm resources but also, can facilitate utilizing them in breeding programs because $F_{1}$ hybrids may be more resistant than either of the parental lines (Clements et al. 2004). Chinese maize germplasm is relatively complex, including both exotic germplasm (the United States and the CIMMTY) and landraces (e.g., SPT). Based on previous reports (Lu et al. 2009; Teng et al. 2004; Wang et al. 2008; Xie et al. 2008; Xu et al. 2018), Chinese germplasm can be largely divided into eight groups (SPT, LRC, PA, PB, Reid, Lancaster, Iodent, and subtropical/tropical).

ANOVA revealed significant differences $(P<0.01)$ in FER resistance among eight germplasm groups in three environments: Shunyi 2017, Xinxiang 2017, and Shunyi 2018 (Fig. 6). Tropical germplasm showed stronger FER resistance than other groups across the four environments. The means of FER severity in Shunyi 2017, Xinxiang 2017, Shunyi 2018, and Xinxiang 2018 were 0.15, 0.12, 0.16, and 0.12 , respectively, for tropical germplasm and $0.22,0.24,0.22$, and 0.29 , respectively, for temperate germplasm (Fig. 6). These findings indicate that tropical germplasm is an important resistance source for
FER, which is line with the previous studies (Chen et al. 2016; Pérez Brito et al. 2001; Small et al. 2012). Among seven temperate germplasm groups, LRC, PA, PB, and Reid had no significant difference in FER severity from tropical germplasm, whereas Iodent and Lancaster germplasm groups were susceptible to FER. Under the three environments (excluding Shunyi 2018), Iodent showed the lowest FER resistance. Iodent belongs to early maturing germplasm, indicating that early flowering is associated with weak FER resistance (Chen et al. 2016). A weak negative correlation was found between FER severity and flowering time (DTT, DTS, and DTA) in the present study (data not shown). As a widely used germplasm group for producing commercial hybrids in China, SPT is relatively susceptible to FER.

The power of association mapping depends on population size, target allele effect, density of markers, LD decay between markers and target alleles, and errors in phenotypic and genotypic data (Yan and Warburton 2011). Because it is limited by overall cost and difficulty in managing large-scale trials, population size needs to be controlled within a reasonable range. For quantitative traits, allele effects are generally small with many genes involved, and bigger population sizes and higher density markers are needed to detect alleles with small effects from many loci. Compared with single SNP analysis, using SNP haplotypes can significantly reduce the number of markers required while also providing higher QTL detection power (Yan and Warburton 2011). Although high-density chips currently available contain tens of thousands or even hundreds of thousands of markers, distribution of markers on chromosomes is still sparse for maize with large genome so that many loci may not be
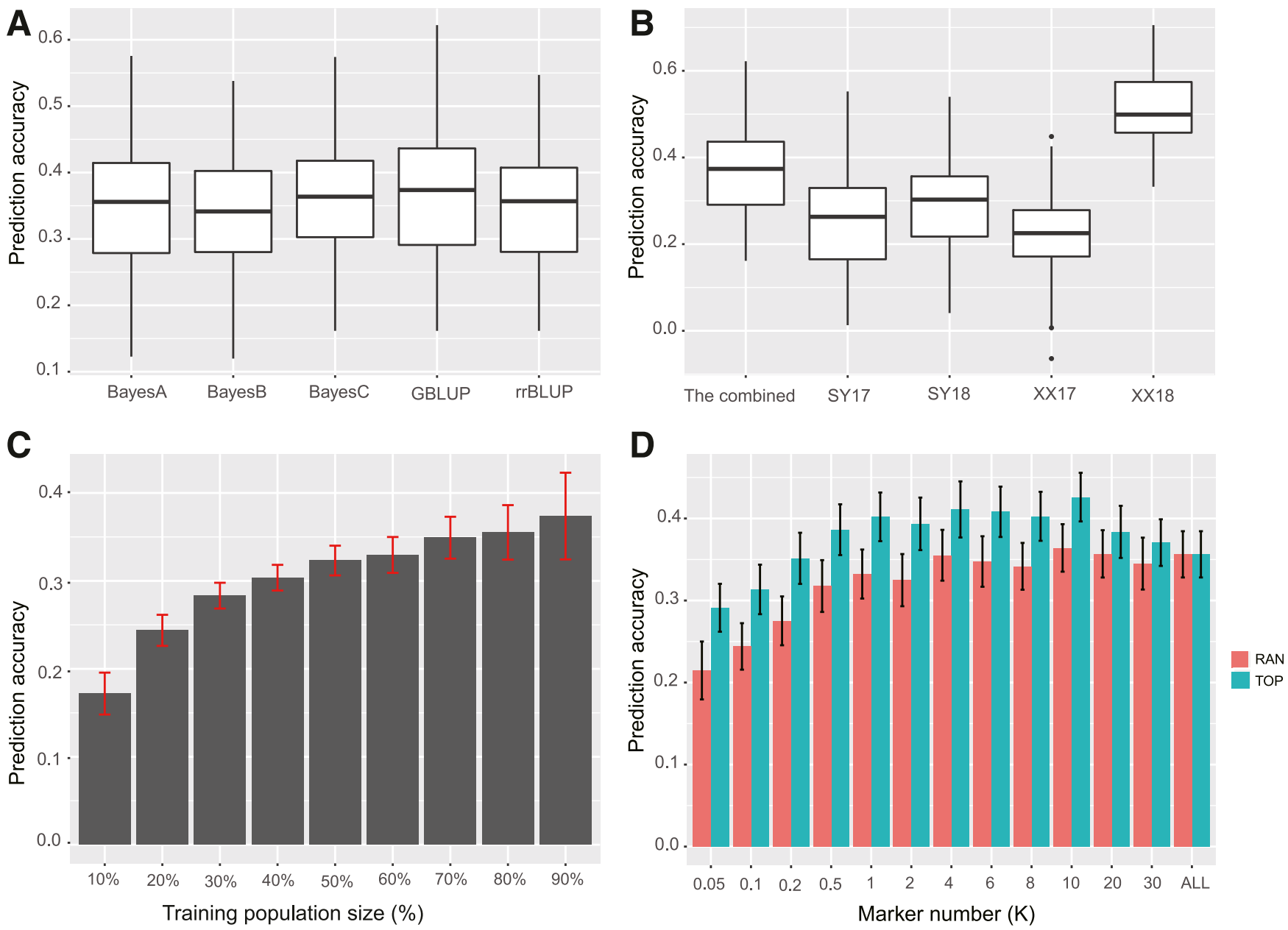

Fig. 5. The comparison of prediction accuracy between $\mathbf{A}$, five genomic selection models, $\mathbf{B}$, different environments, $\mathbf{C}$, training population size, and $\mathbf{D}$, trait-related markers and randomly selected markers. Three Bayesian models are BayesA, BayesB, and BayesC. SY17, XX17, SY18, and XX18 represent Shunyi 2017, Xinxiang 2017, Shunyi 2017, and Xinxiang 2018, respectively. GBLUP, genomic best linear unbiased prediction; RAN, randomly selected marker; rrBLUP, ridge regression-best linear unbiased prediction; TOP, traitrelated marker. 
covered. BSA combined with next generation sequencing cannot only reduce genotyping cost but also, is often more effective in SNP discovery with more accurate allele frequency estimation (Futschik and Schlotterer 2010). In this study, both GWAS and BSA methods were used to identify the loci associated with FER resistance, with some identified loci overlapping.

In the present study, SNPs associated with maize FER resistance on chromosome bins 10.03 and 10.04 were identified by GWAS and BSA, respectively, and the latter has not been reported previously. The locus on bin 10.03 has been reported in two association mappings (Chen et al. 2016; Coan et al. 2018) and one QTL mapping (Ding et al. 2008) containing a large QTL conditioning resistance to multiple maize diseases, including common rust resistance genes rpl and rp5 (Wisser et al. 2006). Therefore, chromosome bin 10.03 is likely to contain important genes related to FER resistance.

There are two kinds of disease resistance in plants: major genecontrolled qualitative resistance and minor gene-controlled quantitative resistance. Maize FER resistance has been proven to be a complex quantitative trait controlled by multiple genes (Chen et al. 2012; Ding et al. 2008; Maschietto et al. 2017; Pérez Brito et al. 2001; Robertson-Hoyt et al. 2006). For quantitative resistance, one of the advantages is that the germplasms with FER resistance are stable and lasting. Therefore, identifying quantitative resistance germplasms is important. Resistance genes widely exist in various varieties of cultivated plants in different regions, especially in wild relatives and landraces. Maize was domesticated from Balsas teosinte that is endemic to the mid- to lowland regions of southwest Mexico (Van Heerwaarden et al. 2011), where tropical environments tend to have a greater diversity of biotic and abiotic stresses than temperate environments. Therefore, selection under these conditions could be responsible for the higher level of FER resistance. As a complex genetic system, no reliable candidate genes for FER resistance have been found through GWAS or linkage analysis, but relatively high heritability indicates that FER is a heritable trait (Pérez Brito et al. 2001). Therefore, it is feasible to make use of the FER-resistant tropical germplasm to improve susceptible temperate germplasm.

For quantitative traits controlled by many genes, the best approach for molecular breeding should shift from MAS to GS (Lanubile et al. 2017), and GS has been considered most promising (Xu et al. 2020). The prediction accuracy of GS is influenced by population size, marker density, heritability, statistical model, population relationship, and the ratio of population sizes between the training and testing sets (Liu et al. 2018; Zhang et al. 2015; Zhao et al. 2012). In the current study, the difference of FER resistance in genomic prediction accuracy among five models was not significant but significant $(P<0.01)$ among four environments, suggesting that prediction accuracy is affected by genotype $\times$ environment interaction. Because a diverse natural population was used, the prediction accuracy for FER resistance was not very high, which is in line with the previous research (Cao et al. 2017; Gowda et al. 2015). The prediction accuracy was improved by using trait-related markers, suggesting that GS should be combined with GWAS. In the future as genotyping cost decreases with the development of new platforms, such as genotyping by target sequencing (Guo et al. 2019), GS will be used more widely for molecular breeding of maize complex traits.
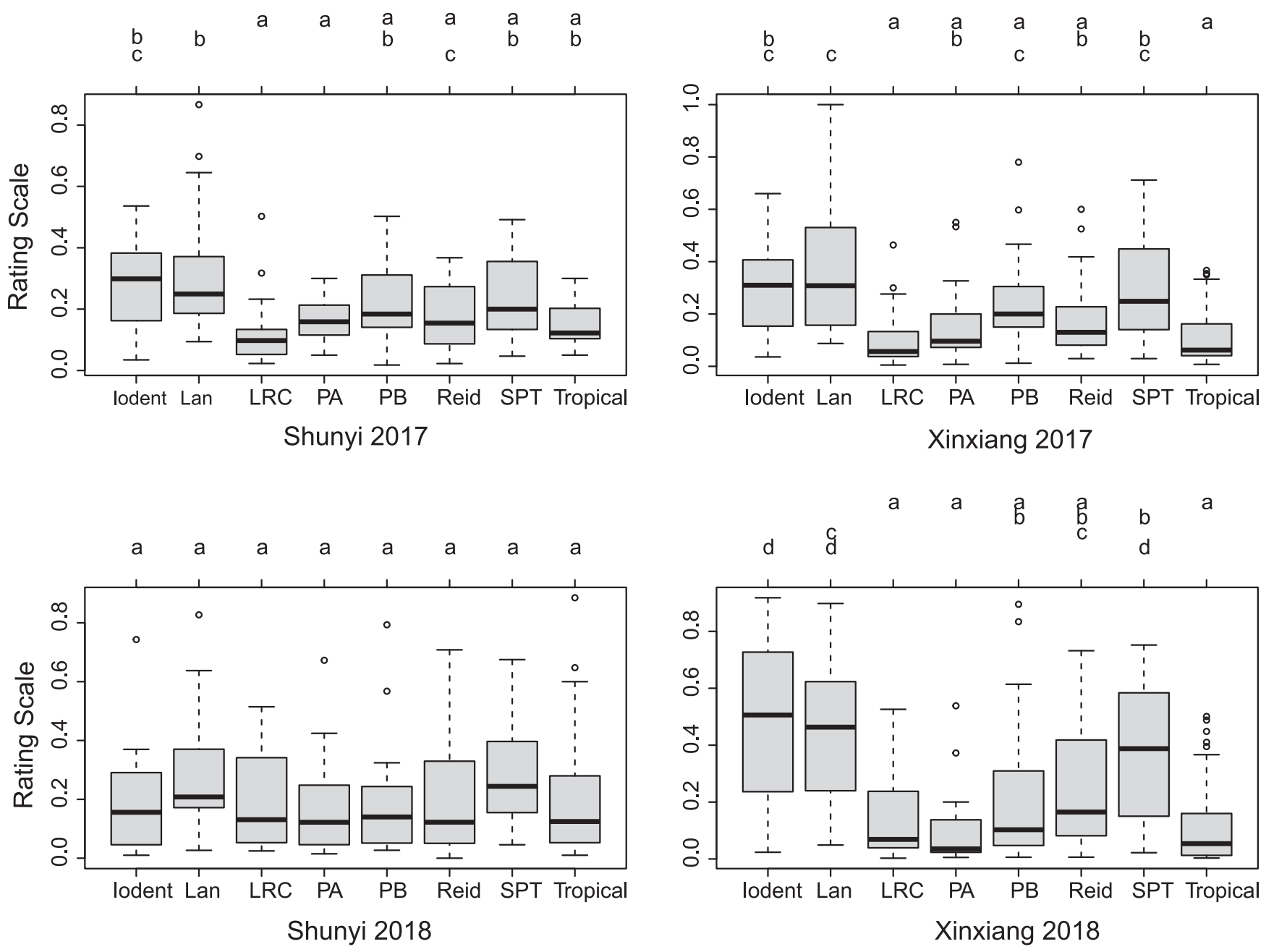

Fig. 6. Significance analysis of resistance to Fusarium ear rot among eight heterotic groups. Lan represents Lancaster. 


\section{Literature Cited}

Balconi, C., Berardo, N., Locatelli, S., Lanzanova, C., Torri, A., and Redaelli, R. 2014. Evaluations of ear rot (Fusarium verticillioides) resistance and fumonisin accumulation in Italian maize inbred lines. Phytopathol. Mediterr. 53:14-26.

Bastide, H., Betancourt, A., Nolte, V., Tobler, R., Stöbe, P., Futschik, A., and Schlötterer, C. 2013. A genome-wide, fine-scale map of natural pigmentation variation in Drosophila melanogaster. PLoS Genet. 9:e1003534.

Bush, B. J., Carson, M. L., Cubeta, M. A., Hagler, W. M., and Payne, G. A. 2004. Infection and fumonisin production by Fusarium verticillioides in developing maize kernels. Phytopathology 94:88-93.

Cao, S., Loladze, A., Yuan, Y., Wu, Y., Zhang, A., Chen, J., Huestis, G., Cao, J., Chaikam, V., Olsen, M., Prasanna, B. M., Vicente, F. S., and Zhang, X. 2017. Genome-wide analysis of tar spot complex resistance in maize using genotyping-by-sequencing SNPs and whole-genome prediction. Plant Genome 10:1-14.

Chen, J., Ding, J., Li, H., Li, Z., Sun, X., Li, J., Sun, X., Li, J., Wang, R., Dai, X., Dong, H., Song, W., and Wu, J. 2012. Detection and verification of quantitative trait loci for resistance to Fusarium ear rot in maize. Mol. Breed. 30:1649-1656.

Chen, J., Shrestha, R., Ding, J., Zheng, H., Mu, C., Wu, J., and Mahuku, G. 2016. Genome-wide association study and QTL mapping reveal genomic loci associated with Fusarium ear rot resistance in tropical maize germplasm. G3 (Bethesda) 6:3803-3815.

CIMMYT. 1998. A complete listing of improved maize germplasm from CIMMYT. Maize Program Special Report. CIMMYT, El Batan, Mexico.

Clements, M. J., Maragos, C. M., Pataky, J. K., and White, D. G. 2004. Sources of resistance to fumonisin accumulation in grain and Fusarium ear and kernel rot of corn. Phytopathology 94:251-260.

Coan, M. M. D., Senhorinho, H. J. C., Pinto, R. B., Scapim, C. A., Tessmann, D. J., Williams, W. P., and Warburton, M. L. 2018. Genome-wide association study of resistance to ear rot by fusarium verticillioides in a tropical field maize and popcorn core collection. Crop Sci. 58:564-578.

Ding, J. Q., Wang, X. M., Chander, S., Yan, J. B., and Li, J. S. 2008. QTL mapping of resistance of resistance to Fusarium ear rot using a RIL population in maize. Mol. Breed. 22:395-403.

Drepper, W. J., and Renfro, B. L. 1990. Comparison of methods for inoculation of ears and stalks of maize with Fusarium moniliforme. Plant Dis. 74:952-956.

Duan, C., Wang, X., Song, F., Sun, S., Zhou, D., and Zhu, Z. 2015. Advances in research on maize resistance to ear rot. Scientia Agric. Sinica 48:2125-2164.

Eller, M. S., Payne, G. A., and Holland, J. A. 2010. Selection for reduced Fusarium ear rot an fumonisin content in advanced backcross maize lines and their topcross hybrids. Crop Sci. 50:2249-2260.

Futschik, A., and Schlotterer, C. 2010. The next generation of molecular markers from massively parallel sequencing of pooled DNA samples. Genetics 186: 207-218.

Giomi, G., Kreff, E., Iglesias, J., Fauguel, C., Fernandez, M., Oviedo, M., and Presello, D. 2016. Quantitative trait loci for Fusarium and Gibberella ear rot resistance in Argentinian maize germplasm. Euphytica 211:287-294.

Goddard, M. E., and Hayes, B. J. 2007. Genomic selection. J. Anim. Breed. Genet. 124:323-330.

Gore, M. A., Chia, J. M., Elshire, R. J., Sun, Q., Ersoz, E. S., Hurwitz, B. L., Peiffer, J. A., McMullen, M. D., Grills, G. S., Ross-Ibarra, J., Ware, D. H., and Buckler, E. S. 2009. A first-generation haplotype map of maize. Science 326:1115-1117.

Görtz, A., Oerke, E. C., Steiner, U., Waalwijk, C., Vries, I., and Dehne, H. W. 2008. Biodiversity of Fusarium species causing ear rot of maize in Germany. Cereal Res. Commun. 36(Supplement 6):617-622.

Gowda, M. B., Makumbi, D. D., Semagn, R. B. K., Mahuku, G., Mahuku, G., Olsen, M. S., Bright, J. M., Beyene, Y., and Prasanna, B. M. 2015. Genomewide association and genomic prediction of resistance to maize lethal necrosis disease in tropical maize germplasm. Theor. Appl. Genet. 128: 1957-1968.

Guo, Z., Wang, H., Tao, J., Ren, Y., Xu, C., Wu, K., Zou, C., Zhang, J., and Xu, Y. 2019. Development of multiple SNP marker panels affordable to breeders through genotyping by target sequencing (GBTS) in maize. Mol. Breed. 39:37.

Han, B., Kang, H. M., and Eskin, E. 2009. Rapid and accurate multiple testing correction and power estimation for millions of correlated markers. PLoS Genet. 5:e1000456.

Jeffers, D. P. 2003. Disease control. Pages 669-716 in: Corn: Origin, History, Technology, and Production, Wiley Series in Crop Science. C. W. Smith, J. Betrán, and E. C. A. Runge, eds. John Wiley \& Sons, Hoboken, NJ.

Lanubile, A., Maschietto, V., Borrelli, V. M., Stagnati, L., Logrieco, A. F., and Marocco, A. 2017. Molecular basis of resistance to Fusarium ear rot in maize. Front. Plant Sci. 8:1774.

Li, H., and Durbin, R. 2009. Fast and accurate short read and alignment with burrows-wheeler transform. Bioinformatics 25:1754-1760.

Li, H., Handsaker, B., Wysoker, A., Fennell, T., Ruan, J., Homer, N., Marth, G., Abecasis, G., and Durbin, R. 2009. The sequence alignment-map format and SAMtools. Bioinformatics 25:2078-2079.

Liu, X., Wang, H., Wang, H., Guo, Z., Xu, X., Liu, J., Wang, S., Li, W. X., Zou, C., Prasanna, B. M., Olsen, M. S., Huang, C., and Xu, Y. 2018. Factors affecting genomic selection revealed by empirical evidence in maize. Crop J. 6:341-352.
Lu, Y., Yan, J., Guimarães, C. T., Taba, S., Hao, Z., Gao, S., Chen, S., Li, J. Zhang, S., Vivek, B. S., Magorokosho, C., Mugo, S., Makumbi, D., Parentoni, S. N., Shah, T., Rong, T., Crouch, J. H., and Xu, Y. 2009. Molecular characterization of global maize breeding germplasm based on genome-wide single nucleotide polymorphisms. Theor. Appl. Genet. 120: 93-115.

Maschietto, V., Colombi, C., Pirona, R., Pea, G., Strozzi, F., Marocco, A., Rossini, L., and Lanubile, A. 2017. QTL mapping and candidate genes for resistance to Fusarium ear rot and fumonisin contamination in maize. BMC Plant Biol. 17:20.

McKenna, A., Hanna, M., Banks, E., Sivachenko, A., Cibulskis, K., Kernytsky, A. Garimella, K., Altshuler, D., Gabriel, S., Daly, M., and DePristo, M. A. 2010. The genome analysis toolkit: A MapReduce framework for analysis nextgeneration DNA sequencing data. Genome Res. 20:1297-1303.

Mesterházy, Á., Lemmens, M., and Reid, L. M. 2012. Breeding for resistance to ear rots caused by Fusarium spp. in maize-a review. Plant Breed. 131:1-19.

Meuwissen, T. H. E., Hayes, B. J., and Goddard, M. E. 2001. Prediction of total genetic value using genome-wide dense marker maps. Genetics 157:1819-1829.

Munkvold, G. P. 2003. Epidemiology of Fusarium diseases and their mycotoxins in maize ears. Eur. J. Plant Pathol. 109:705-713.

Munkvold, G. P., and Desjardins, A. E. 1997. Fumonisins in maize: Can we reduce their occurrence? Plant Dis. 81:556-565.

Pérez Brito, D., Jeffers, D. P., González de León, D., Khairallah, M. M., Cortés, C. M., Velázquez, C. G., Azpíroz, S., and Srinivasan, G. 2001. QTL mapping of Fusarium moniliforme ear rot resistance in highland maize, Mexico. Agrociencia 35:181-196.

Placinta, C. M., D’Mello, J. P. F., and Macdonald, A. M. C. 1999. A review of worldwide contamination of cereal grain and animal feed with Fusarium mycotoxins. Anim. Feed Sci. Technol. 78:21-37.

R Core Team. 2015. R: A language and environment for statistical computing. R Foundation for Statistical Computing, Vienna, Austria. https://www.R-project. org/.

Robertson-Hoyt, L. A., Jines, M. P., Balint-Kurti, P. J., Kleinschmidt, C. E., White, D. G., Payne, G. A., Maragos, C. M., and Holland, J. B. 2006. QTL mapping for Fusarium ear rot and Fumonisin contamination resistance in two maize populations. Crop Sci. 46:1734-1743.

Rose, L. J., Mouton, M., Beukes, I., Flett, B. C., van der Vyver, C., and Viljoen, A. 2016. Multi-environment evaluation of maize inbred lines for resistance to Fusarium ear rot and fumonisins. Plant Dis. 100:2134-2144.

Rutkoski, J. E., Poland, J. A., Singh, R. P., Huerta-Espino, J., Bhavani, S., Barbier H., Rouse, M. N., Jannink, J. L., and Sorrells, M. E. 2014. Genomic selection for quantitative adult plant stem rust resistance in wheat. Plant Genome 7:1-10.

Schlötterer, C., Tobler, R., Kofler, R., and Nolte, V. 2014. Sequencing pools of individuals-mining genome-wide polymorphism data without big funding. Nat. Rev. Genet. 15:749-763.

Small, I. M., Flett, B. C., Marasas, W. F. O., McLeod, A., and Viljoen, A. 2012. Use of resistance elicitors to reduce Fusarium ear rot and fumonisin accumulation in maize. Crop Prot. 41:10-16.

Teng, W. T., Cao, Q. S., Chen, Y. H., Liu, X. H., Men, S. D., Jing, X. Q., and Li, J. S. 2004. Analysis of maize heterotic groups and patterns during past decade in China. Agric. Sci. China 3:481-489.

van Heerwaarden, J., Doebley, J., Briggs, W. H., Glaubitz, J. C., Goodman, M. M. de Jesus Sanchez Gonzalez, J., and Ross-Ibarra, J. 2011. Genetic signals of origin, spread, and introgression in a large sample of maize landraces. Proc. Natl. Acad. Sci. USA 108:1088-1092.

Wang, R., Yu, Y., Zhao, J., Shi, Y., Song, Y., Wang, T., and Li, Y. 2008 Population structure and linkage disequilibrium of a mini core set of maize inbred lines in China. Theor. Appl. Genet. 117:1141-1153.

Wisser, R. J., Balint-Kurti, P. J., and Nelson, R. J. 2006. The genetic architecture of disease resistance in maize: A synthesis of published studies. Phytopathology 96:120-129.

Xie, C. X., Warburton, M., Li, M. S., Li, X. H., Xiao, M. J., Hao, Z. F., Zhao, Q., and Zhang, S. H. 2008. An analysis of population structure and linkage disequilibrium using multilocus data in 187 maize inbred lines. Mol. Breed. 21:407-418.

Xu, C., Ren, Y., Jian, Y., Guo, Z., Zhang, Y., Xie, C., Fu, J., Wang, H., Wang, G., Xu, Y., Li, P., and Zou, C. 2017. Development of a maize 55K SNP array with improved genome coverage for molecular breeding. Mol. Breed. 37:20.

Xu, C., Zhang, H., Sun, J., Guo, Z., Zou, C., Li, W. X., Xie, C., Huang, C., Xu, R., Liao, H., Wang, J., Xu, X., Wang, S., and Xu, Y. 2018. Genome-wide association study dissects yield components associated with low-phosphorus stress tolerance in maize. Theor. Appl. Genet. 131:1699-1714.

Xu, Y., Liu, X., Fu, J., Wang, H., Wang, J., Huang, C., Prasanna, B. M., Olsen, M. S., Wang, G., and Zhang, A. 2020. Enhancing genetic gain through genomic selection: From livestock to plants. Plant Comm. 1:100005.

Yan, J., and Warburton, M. L. 2011. Association mapping for enhancing maize (Zea mays L.) genetic improvement. Crop Sci. 51:433-449.

Yang, J., Manolio, T. A., Pasquale, L. R., Boerwinkle, E., Caporaso, N., Cunningham, J. M., de Andrade, M., Feenstra, B., Feingold, E., Hayes, M. G., Hill, W. G., Landi, M. T., Alonso, A., Lettre, G., Lin, P., Ling, H., Lowa, W., Mathias, R. A., Melbye, M., Pugh, E., Cornells, M. C., Weir, B. S., Goddard, M. E., and Visscher, P. M. 2011. Genome partitioning of genetic variation for complex traits using common SNPs. Nat. Genet. 43:519-525. 
Yu, J., and Buckler, E. S. 2006. Genetic association mapping and genome organization of maize. Curr. Opin. Biotechnol. 17:155-160.

Zhang, X., Pérez-RodrÍguez, P., Semagn, K., Beyene, Y., Babu, R., López-Curz, M. A., San Vicente, F., Olsen, M., Buckler, E., Jannink, J. L., Prasanna, B. M., and Crossa, J. 2015. Genomic prediction in biparental tropical maize populations in water-stressed and well-watered environments using lowdensity and GBS SNPs. Heredity 114:291-299.

Zhao, Y., Gowda, M., Liu, W., Würschum, T., Maurer, H. P., Longin, F. H., Ranc, N., and Reif, J. C. 2012. Accuracy of genomic selection in European maize elite breeding populations. Theor. Appl. Genet. 124:769-776.
Zila, C. T., Ogut, F., Romay, M. C., Gardner, C. A., Buckler, E. S., and Holland, J. B. 2014. Genome-wide association study of Fusarium ear rot disease in the U.S.A maize inbred line collection. BMC Plant Biol. 14:372.

Zila, C. T., Samayoa, L. F., Santiago, R., Butrón, A., and Holland, J. B. 2013. A genome-wide association study reveals genes associated with Fusarium ear ro resistance in a maize core diversity panel. Genes Genom Genet. 3:2095-2104

Zou, C., Wang, P., and Xu, Y. 2016. Bulked sample analysis in genetics, genomics and crop improvement. Plant Biotechnol J. 14:1941-1955.

Zummo, N., and Scott, G. E. 1990. Cob and kernel infected by Aspergillus flavus and Fusarium moniliforme in inoculated, field-grown maize ears. Plant Dis. 74:627-631. 